Article

\title{
Interactive Buckling of Steel LC-Beams Under Bending
}

\author{
Zbigniew Kolakowski *(D) and Jacek Jankowski \\ Lodz University of Technology, 90-924 Lodz, Poland; jacek.jankowski@p.lodz.pl \\ * Correspondence: zbigniew.kolakowski@p.lodz.pl; Tel.: +48-42-631-22-15
}

Received: 3 April 2019; Accepted: 30 April 2019; Published: 3 May 2019

\begin{abstract}
The present paper deals with the interactive buckling of thin-walled lipped channel (LC) beams under the bending moment in the web plane when the shear lag phenomenon and distortional deformations are taken into account. A plate model (2D) was adopted for LC beams. The structures were assumed to be simply supported at the ends. A modal method of solution to the interactive buckling problem within Koiter's asymptotic theory, using the semi-analytical method (SAM) and the transition matrix method, was applied. LC-beams, from short through medium-long via long to very long beams, were considered. The paper focuses on the influence of the secondary global buckling mode on the load carrying capacity for the steel LC-beams under bending.
\end{abstract}

Keywords: lip channel; bending; interactive buckling; distortional-lateral mode; different lengths

\section{Introduction}

Thin-walled cold-formed steel (CFS) members (columns, beams, and beam-columns) are widely used in the construction industry. C-section and LC-section (i.e., lipped channel) beams are basic structural elements that are primarily subject to bending. A capacity for resistant loads in thin-walled beams is limited not just by their strength, but first of all due to their stability.

The numerical methods often applied in nonlinear analysis of stability and load carrying capacity are as follows: finite strip method (FSM), finite element method (FEM), and generalized beam theory (GBT).

Mode decomposition of thin-walled columns and beams with different cross-sections subjected to various loadings based on GBT has been analyzed in Reference [1]. References [2,3] deal with the constrained finite element method (cFEM) employed for the buckling analysis of columns with opened cross-sections. A new method with a modal decomposition feature, the so-called constrained finite element method (cFEM) is presented in Reference [4]. The method can be applied to analyze a wide range of thin-walled members, including members with holes and varying cross-sections and stiffened plates.

FSM is commonly used for nonlinear analysis of elastic stability due to its very high numerical capability, low computational costs and easy implementation in thin-walled elements. The method is limited mainly to simple geometries and boundary conditions. It has resulted in an introduction of numerous new FSM variants or expansions.

In FEM, two kinds of analyses are usually conducted, namely for (i) linear elastic buckling to enable determination of critical loads and the corresponding buckling modes, i.e., an eigenproblem, (ii) nonlinear post-buckling analysis to determine the performance curve in the full range of loading and/or load carrying capacity. In addition, the FEM model is affected by epistemic uncertainties.

The influence of distortional, global, and local buckling modes, and their combination, on post-buckling behavior is widely investigated by Martins, Camotim, Gonçalves, and Dinis $[5,6]$. They and used their modification (GBTUL-2.0) [5] and employed existing tools (GBT and DSM) [6]. A semi-analytic method based on Koiter's theory of the compressed thin-walled structure with cross-section deformation modes is analyzed in Reference [7]. Composite C-beams subjected to the 
bending moment have been analyzed in References [8], among others. The development in the theory of interactive buckling of thin-walled structures is discussed in Reference [9]. Observation of the post-buckling behavior of beams during experimental investigations of channel-section beams subjected to pure bending [10] inspired the research presented here. Moreover, another interpretation of the obtained results has been adopted. Reference [10] that dealt with post-buckling behavior of short beams with channel-section, subjected to pure bending, were the basis to carry out the research presented in this paper. In References [11,12], the nonlinear problem of stability has been solved using the semi-analytical method (SAM) based on Koiter's nonlinear theory with Byskov-Hutchinson interactive buckling approach. An interaction between two or three buckling modes (i.e., two- and three-mode approach) has been analyzed, whereas in Reference [13] numerous buckling modes were considered. The effect of various lengths (from the short ones through medium-long to long beams) of C-beams subjected to the bending moment, with linearly variable stresses in the web plane, on the interactive buckling and load carrying capacity using semi analytical method (SAM) [11,12] was investigated. The present paper is a continuation of Reference [13], focusing on steel bending LC-beams to confirm the generality of the results obtained in Reference [13] and to specify the length of the beam for which the influence of the secondary mode of buckling on load carrying capacity appears in the range of the most dangerous case. A literature review and the theoretical foundation are presented in detail in Reference [13].

The post-buckling behavior of beams during experimental investigations of channel-section beams subjected to pure bending was described in Reference [14]. Moreover, the influence of the distortional-lateral buckling mode on the interactive buckling of thin-walled short channels with imperfections subjected to the major-axis bending moment was analyzed. With the GBT it is possible to define contributions of different buckling modes in the full range of structure loading, which facilitates understanding the phenomena occurring during complex coupled buckling.

Moreover, composite C-beams subjected to the bending moment have been analyzed in Reference [15], among others.

A distortional failure of steel beams simply supported under uniform bending with the direct strength method (DSM) is presented in Reference [16]. The influence of distortional, global, and local buckling modes, and their combination, on post-buckling behavior is widely investigated by Martins, Camotim, Gonçalves, and Dinis [17,18]. In Reference [19], the interactive buckling of lipped channel (LC) beams freely supported at both ends and subject to uniform major-axis bending was analyzed with the generalized beam theory (GBT). Special attention was paid to the interaction of very numerous simple buckling modes, and, in particular, to the distortional-global mode effect. Moreover, the influence of distortional, global, and local buckling modes, and their combination, on post-buckling behavior is widely investigated in References [20,21].

In this study, an analysis of LC beams with the same dimensions as in Reference [19] has been conducted. In wide experimental investigations and the numerical analysis of steel C-beams [22,23], particular attention was drawn to the distortional-global interaction buckling. Post-buckling behavior of cold-formed channels axially compressed or subjected to pure bending was analyzed in Reference [24]. In Reference [25], the buckling behavior and imperfection sensitivity of thin steel cylindrical shells under pure bending, with a focus range of slenderness, were presented.

The fire-resistant design of steel columns used in prefabricated modular construction was described in References [26,27].

Modal analysis of interactive buckling enables an easier insight into the phenomena occurring during the interactions of global, local, and distortional buckling modes. Moreover, it allows an interpretation of complex interactions between individual buckling modes.

In the authors' opinion, there is a lack of work devoted to the influence of the secondary global distortional-lateral mode on the interactive buckling and assessment of the load carrying capacity for LC-beams of various lengths. The paper attempts to assess for what length of beam this impact is the most important. In the authors' opinion, the literature includes adequate information about the 
influence of the secondary global distortional-lateral mode on the interactive buckling and assessment of the load carrying capacity of LC-beams of various lengths. The aim of this investigation is to determine what values of length of the beam influences on the load carrying capacity.

In the present study, Lagrange's description, full Green's strain tensor for thin-walled plates and second Piola-Kirchhoff's stress tensor, and the exact transition matrix method and the numerical method of the transition matrix using Godunov's orthogonalization are used. The shear lag phenomenon, an effect of cross-sectional distortions, as well as coupled conditions between all the walls of structures are included. The most important advantage of this method is that a complete range of behavior of thin-walled structures can be described [14].

The coupled buckling of thin-walled steel LC-beams under bending in the web plane from short ones through medium-long to long beams is analyzed here.

\section{Formulation of the Problem}

Prismatic thin-walled steel (i.e., isotropic) beams built of plates connected along longitudinal edges and under the uniform major-axis bending moment were considered. The beams were simply supported at their ends $[13,14]$. In order to account for all modes of global, local, and coupled buckling, a plate model (i.e., 2D) of thin-walled structures was applied. Moreover, it was assumed that the material of the structure is obeyed Hooke's law. Details can be found in References $[13,14]$ or see Appendix A.

\section{Analysis of the Calculations Results}

Detailed numerical calculations for interactive buckling were conducted for three steel LC-section beams of the cross-section dimensions identical to those in Reference [19]. The beam geometrical dimensions under consideration together with the assumed notations are presented in Figure 1 and Table 1. The ratios of the main central moments of inertia $I_{\max } / I_{\text {min }}$, which range from 3.6 to 6.0 , are included there as well.

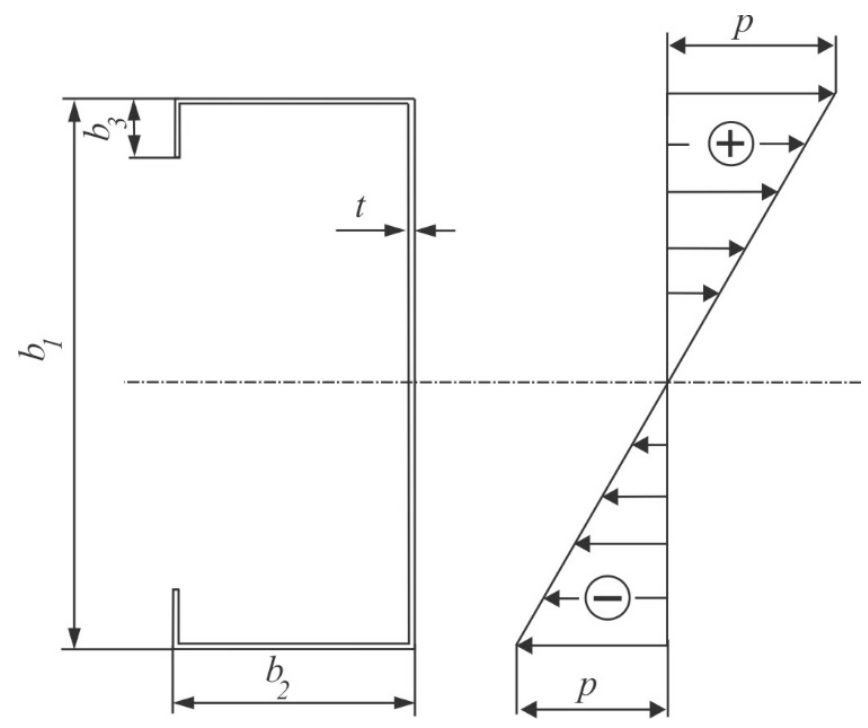

Figure 1. Cross-section of the lip channel.

Table 1. Geometrical dimensions considered lipped channel (LC)-beams.

\begin{tabular}{cccccc}
\hline \multirow{2}{*}{ Type of the Beam } & $\boldsymbol{b}_{\mathbf{1}}$ & $\boldsymbol{b}_{\mathbf{2}}$ & $\boldsymbol{b}_{\mathbf{3}}$ & $\boldsymbol{t}$ & $\boldsymbol{I}_{\max } / \boldsymbol{I}_{\min }$ \\
\cline { 2 - 6 } & {$[\mathbf{m m}]$} & {$[\mathbf{m m}]$} & {$[\mathbf{m m}]$} & {$[\mathbf{m m}]$} & - \\
\hline LC-1 & 125 & 75 & 10 & 3 & 3.67 \\
\hline LC-2 & 190 & 90 & 10 & 3.08 & 5.94 \\
\hline LC-3 & 175 & 100 & 13 & 3.6 & 4.04 \\
\hline
\end{tabular}


The following material constants: $E=210 \mathrm{GPa}, v=0.3$ were assumed for the steel lip channels [19]. In the pre-buckling state, the beams were subjected to linearly variable loading (Figure 1) resulting in bending in the web plane (i.e., the upper flange was tensioned, the lower one was compressed).

\subsection{Example of LC-1 Beams}

In this example, lip channel LC-1 beams of the dimensions listed in Table 1 were analyzed. Alternations in values of the critical moment $M_{r}$ as a function of the buckling half-wavelength $L_{b}$ in a wide variability range $100 \leq L_{b} \leq 10,000 \mathrm{~mm}$ are presented in Figure 2 .

LC-1

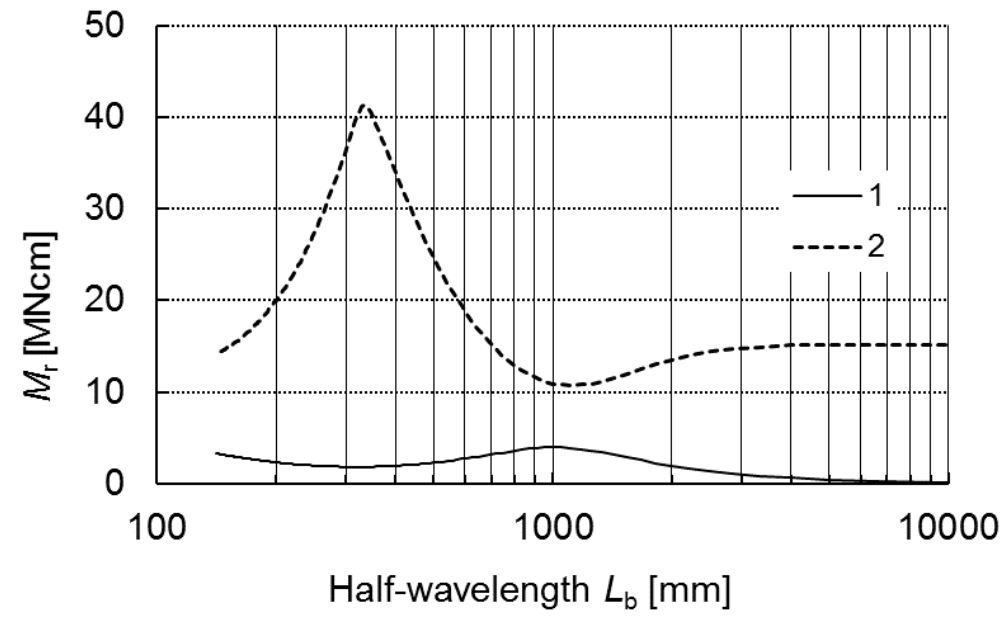

Figure 2. Buckling moments $M_{r}$ as a function of the buckling half-wavelength $L_{b}$ for LC-1.

The lower curve (denoted as curve 1) corresponds to the lowest values of buckling loads, often referred to as primary buckling loads. The upper curve (marked as curve 2) refers to higher critical values, which can be called secondary buckling loads.

The value of the critical moment $M_{r}$ (curve 1 ) for the variability range under consideration attains its maximum at $L_{b}=100 \mathrm{~mm}$ and the minimum at $L_{b} \approx 350 \mathrm{~mm}$, and then it grows monotonously up to $L_{b} \approx 1000 \mathrm{~mm}$, where it reaches the local maximum. Within the range $1000 \leq L_{b} \leq 10,000 \mathrm{~mm}$, values of the moment decrease monotonously. The critical values corresponding to curve 2 grow in the range 100 $\leq L_{b} \leq 350 \mathrm{~mm}$ and attain the maximal value at $L_{b} \approx 350 \mathrm{~mm}$. Next, in the range $350 \leq L_{b} \leq 1000 \mathrm{~mm}$, they decrease drastically to attain the minimal value for $L_{b} \approx 1000 \mathrm{~mm}$. Within the range $1000 \leq L_{b}$ $\leq 4000 \mathrm{~mm}$, curve 2 grows slowly, and at $L_{b}>4000 \mathrm{~mm}$, it is constant in practice. While comparing curves 1 and 2 , one can state that for $L_{b} \approx 350 \mathrm{~mm}$ the lower curve attains its minimum, whereas the upper one attains its maximum, and then at $L_{b} \approx 1000 \mathrm{~mm}$, an opposite relation takes place. At $L_{b}>$ $4000 \mathrm{~mm}$, the drop gradient of curve 1 is significantly lower than for $1000 \leq L_{b} \leq 4000 \mathrm{~mm}$, whereas the values corresponding to curve 2 are actually constant.

In Table 2, critical values of the moments $M_{r}$ for the LC-1 subject to bending for selected four values of the total length $L$ are presented. The following index notations are introduced: 1 -the lowest value of the buckling moment corresponding to the local buckling mode for $m \neq 1,2$-the value of the primary global buckling mode for $m=1$ (curve 1 in Figure 2), 3-the value of the secondary global buckling mode for $m=1$ (curve 2 in Figure 2). For the local moment $M_{1}$, a number of half-waves $m$ along the longitudinal direction is quoted additionally in the brackets. 
Table 2. The buckling moments $M_{r}$ with the corresponding number of half-waves $m$ of buckling along the longitudinal direction of the LC- 1 and the dimensionless ratio of load carrying capacities $M_{s} / M_{\text {min }}$ for different lengths $L$.

\begin{tabular}{cccccc}
\hline $\boldsymbol{L}$ & $\boldsymbol{M}_{\mathbf{1}}$ & $\boldsymbol{M}_{\mathbf{2}}$ & $\boldsymbol{M}_{\mathbf{3}}$ & $\boldsymbol{M}_{\boldsymbol{s} \mathbf{1}} / \boldsymbol{M}_{\min }$ & $\boldsymbol{M}_{\boldsymbol{s} \mathbf{2}} / \boldsymbol{M}_{\min }$ \\
\hline $\mathbf{m m}$ & $\mathbf{M N c m}$ & $\mathbf{M N c m}$ & $\mathbf{M N c m}$ & - & - \\
\hline 2050 & $1.575(6)$ & 1.587 & 11.64 & 0.675 & 0.680 \\
\hline 1500 & $1.574(5)$ & 2.525 & 10.13 & 0.773 & 0.791 \\
\hline 500 & $1.683(2)$ & 1.962 & 21.07 & 0.768 & - \\
\hline 250 & $1.683(1)$ & - & 23.42 & - & - \\
\hline
\end{tabular}

For the lengths of LC-1 under consideration, the values of the critical moment $M_{1}$ do not alter significantly (less than $10 \%$ ). The values of $M_{3}$ are at least sixfold higher than $M_{1}$. At the length $L \approx$ $2050 \mathrm{~mm}$, the value $M_{1} \approx M_{2}$, whereas, at $L=1500 \mathrm{~mm}$, we have $M_{2} / M_{1}=1.6$, and for $L=500 \mathrm{~mm}$ it is $M_{2} / M_{1}=1.16$. At the length $L=250 \mathrm{~mm}$, the lowest critical value was attained for the local mode $M_{1}$ and for one buckling half-wave $(m=1)$. Therefore, the critical value $M_{2}$ corresponding to the global mode was not given. The value $M_{3}$ is almost 14 times higher than $M_{1}$ and also occurs for $m=1$.

In Figure $3 \mathrm{a}-\mathrm{d}$, for the lengths of LC-1 beams considered in Table 2, the buckling modes corresponding to the three modes under analysis, except $L=250 \mathrm{~mm}$, for which only two modes (i.e., mode 1 and mode 3 ) are considered, are shown.

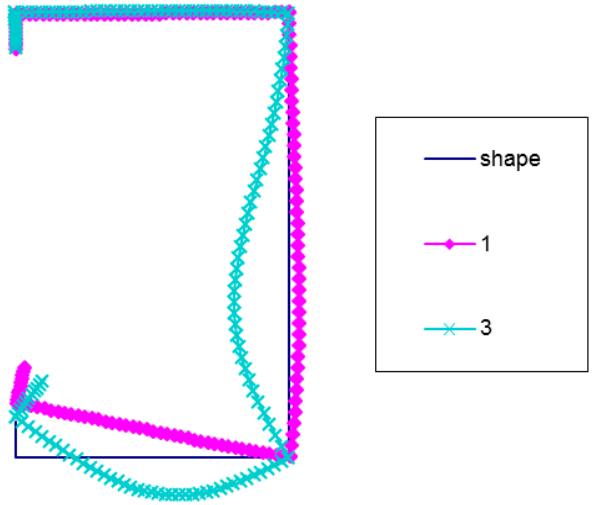

(a)

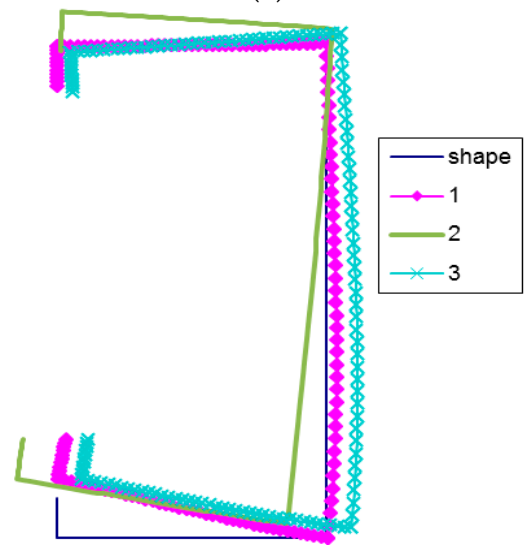

(c)

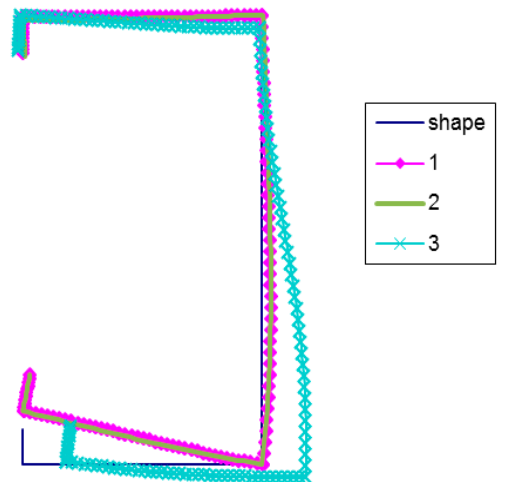

(b)

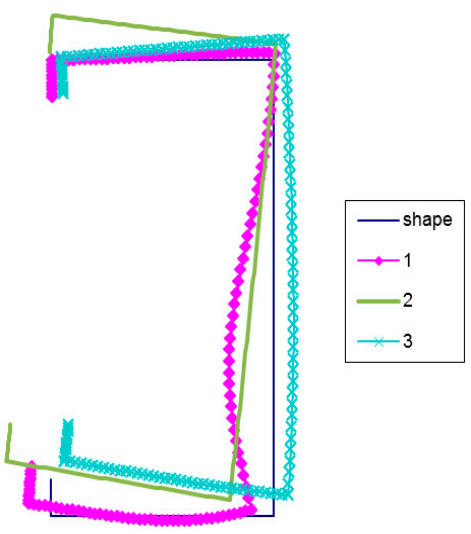

(d)

Figure 3. (a) Buckling modes for LC-1 beam lengths $L=200 \mathrm{~mm}$, (b) Buckling modes for LC-1 beam lengths $L=500 \mathrm{~mm}$, (c) Buckling modes for LC-1 beam lengths $L=1500 \mathrm{~mm}$, (d) Buckling modes for LC-1 beam lengths $L=2050 \mathrm{~mm}$. 
For the three shortest lengths, the local mode $1(r=1)$ is the same (Figure 3a-c). The upper corner connecting the flange under tension with the web and the edge reinforcement does not displace practically. The maximal deflection corresponds to the compressed flange corner with the edge reinforcement. At the length $L=2050 \mathrm{~mm}$ (Figure 3d), the local mode is different. The maximal deflection occurs for the lower part of the web under compression, and both corners of the compressed flange displace. Local modes correspond to distortional-local buckling modes.

At $L=500 \mathrm{~mm}$ (Figure 3b), the lowest global mode (at $r=2$ ) is identical to the local mode. For the lengths $L=1500 \mathrm{~mm}$ and $L=2050 \mathrm{~mm}$ (Figure 3c,d), the global mode represents distortional-lateral buckling because right angles are not maintained in the corners of cross-sections of the elements under compression. The secondary global mode $(r=3)$ is subject to alternations with an increase in the length of LC-1. For $L=200 \mathrm{~mm}$, the maximal deflection occurs for the web. Only the corner connecting the lower edge reinforcement with the flange under compression displaces (Figure 3a). For other lengths, the lower corner connecting the flange with the web also displaces and there is a slight displacement of the corner connecting the web with the flange under compression. At $L=500 \mathrm{~mm}$ (Figure $3 b$ ), the maximal displacement corresponds to lower corners, whereas, at $L=1500 \mathrm{~mm}$ and $L=2050 \mathrm{~mm}$ (Figure 3c,d), the modes differ slightly. The secondary global modes correspond more to the distortional-global modes than the distortional-lateral ones.

In the nonlinear analysis of interactive buckling, the signs of complex absolute values of imperfections of each mode were selected in the safest way, i.e., to attain the lowest value of the limit load carrying capacity $M_{s}$ [11-14] in (A4). For actual LC-section beams, post-buckling equilibrium paths were determined on the assumption in (A4) that $\zeta_{1}^{*}=|0.1|, \zeta_{2}^{*}=|1.0|, \zeta_{3}^{*}=|1.0|$.

For the lengths $L$ under consideration, Table 2 also lists values of the limit load carrying capacity referring to the lowest value of the critical moment $M_{\min }=M_{1}$, and accounts for $M_{s 1} / M_{\min }$ for a three-mode approach (i.e., $J=3$ in (A4)) and $M_{s 2} / M_{\min }$ for a two-mode approach (i.e., $J=2$ ). At $L=250 \mathrm{~mm}$, due to the fact that both modes occur for $m=1$, it was assumed on the contrary that $\zeta_{3}^{*}=|0.1|$. For this length, interaction between buckling modes (denoted by indices $r=1$ and $r=3$ ) does not take place within the loading range $M / M_{\min }$ under analysis.

In Figure 4, on the basis of Equation (A6), a plot of $M / M_{\min }$ versus the angle $\alpha / \alpha_{\min }$ is presented. Curve 1 corresponds to a one-mode analysis, that is to say, when only the mode $J=r=1$ is considered, whereas curve 2 corresponds to a two-mode analysis for $J=2$ (for $r=1$ and $r=3$ ).

LC- $1 \quad L=200 \mathrm{~mm}$

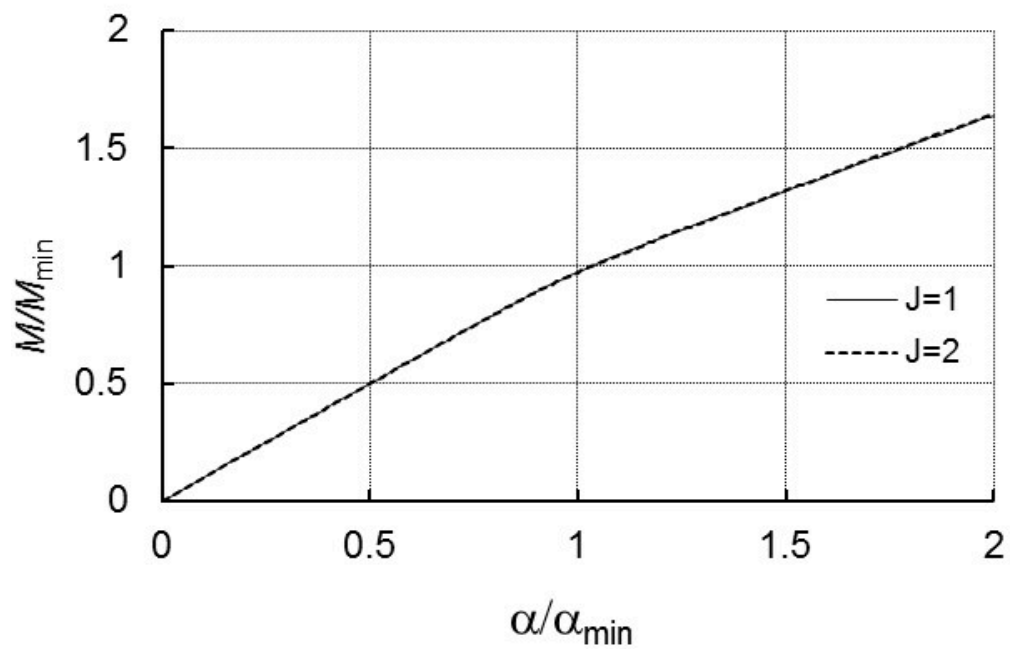

Figure 4. $M / M_{\min }$ as a function of $\alpha / \alpha_{\min }$ for $L C-1$ and $L=200 \mathrm{~mm}$. 
These curves overlap in the range of variability of $M / M_{\text {min }}$ under analysis. At $L=500 \mathrm{~mm}$ (Table 2 and Figure 5), when the interaction of the three modes is taken into account, we have the limit value of $M_{s 1} / M_{\text {min }}$, whereas, for an interaction of two modes (i.e., $J=2$ for $r=1$ and $r=2$ ), the theoretical limit load carrying capacity was not obtained.

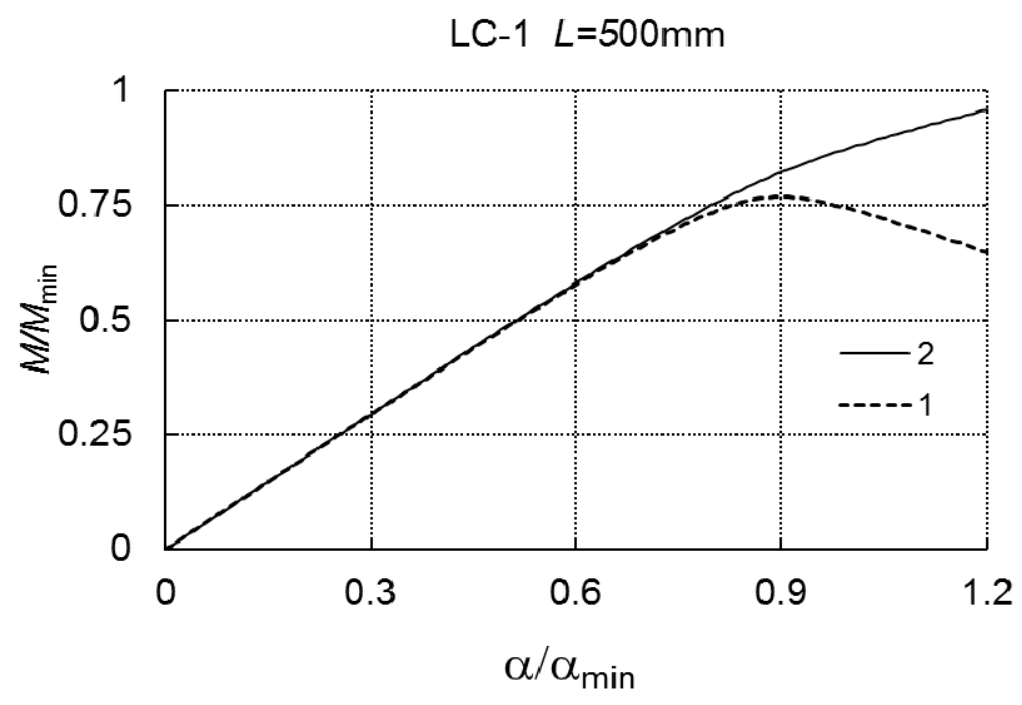

Figure 5. $M / M_{\min }$ as a function of $\alpha / \alpha_{\min }$ for LC-1 and $L=500 \mathrm{~mm}$.

As shown in Figure 5, curve 1 corresponds to the interaction of three modes, whereas curve 2 corresponds to the interaction of two modes, respectively. For $L=1500 \mathrm{~mm}, M_{s 1} / M_{\text {min }}$ is approximately $2 \%$ lower than for $M_{s 2} / M_{\text {min }}$. The lowest values of load carrying capacity were attained at $L=2050 \mathrm{~mm}$ and they are practically the same for the two- and three-mode approach.

The strongest interaction of the local mode $(r=1)$ with the global one $(r=2)$ occurs for the case when the critical loads are close to each other, i.e., when the relationship $0.8 \leq M_{2} M_{1} \leq 1.2$ holds. When $M_{2} / M_{1} \approx 1$, as known from the literature, the load carrying capacity often satisfies the relation $0.6 \leq M_{s} M_{1} \leq 0.7$. For $L=2050 \mathrm{~mm}, M_{2} / M_{1}=1.007$ and $M_{s 1} / M_{1} \approx 0.675$ occur and, for $L=1500 \mathrm{~mm}$, $M_{2} / M_{1}=1.60$ and $M_{s 1} / M_{1}=0.773$ occur, correspondingly, whereas, for $L=500 \mathrm{~mm}, M_{2} / M_{1}=1.16$ and $M_{s 1} / M_{1} \approx 0.768$ occur, respectively. As can be expected, an interaction of three modes yields lower values of the load carrying capacity than an interaction of two modes.

An interaction of buckling modes [11,12] takes place via the coefficients of cubic terms $\bar{a}_{p q r} \zeta_{p} \zeta_{q} \zeta_{r}$ in the expression for total potential energy (A3). Thus, values of the coefficients $\bar{a}_{p q r}$ for all lengths $L$ under study were analyzed. For a short beam of $L=250 \mathrm{~mm}$, the terms including the coefficients $\zeta_{1}^{2} \zeta_{3}$ in (A3) are very low and buckling can be treated as uncoupled (i.e., one-mode) for the loads $M / M_{\text {min }}$ under consideration. It is also due to very considerable differences in values of critical loads, because $M_{3} / M_{1} \approx 14$. At $L=500 \mathrm{~mm}$, the terms $\zeta_{1}^{2} \zeta_{3}, \zeta_{2}^{2} \zeta_{3}$ decide the interaction, whereas, for $L=1500 \mathrm{~mm}$ and $L=2050 \mathrm{~mm}$, these are the terms $\zeta_{1}^{2} \zeta_{2}, \zeta_{1}^{2} \zeta_{3}$.

In Reference [19], local imperfections were taken as in the present work, whereas global imperfections were assumed for selected buckling modes, and their level was close to that assumed here. In Reference [19], for various global imperfections and at $L=2050 \mathrm{~mm}, M_{s} / M_{\min }=0.864$ was attained, and, in this work, $M_{s 1} / M_{\min }=0.675$ was attained.

\subsection{Example of LC-2 Beams}

The geometrical dimensions of the LC-2 beam are listed in Table 2. Figure 6 shows a change in the critical bending moment $M_{r}[\mathrm{MNcm}]$ as a function of the buckling half-wavelength $L_{b}$ in the range 100 $\leq L_{b} \leq 10,000 \mathrm{~mm}$. 
LC-2

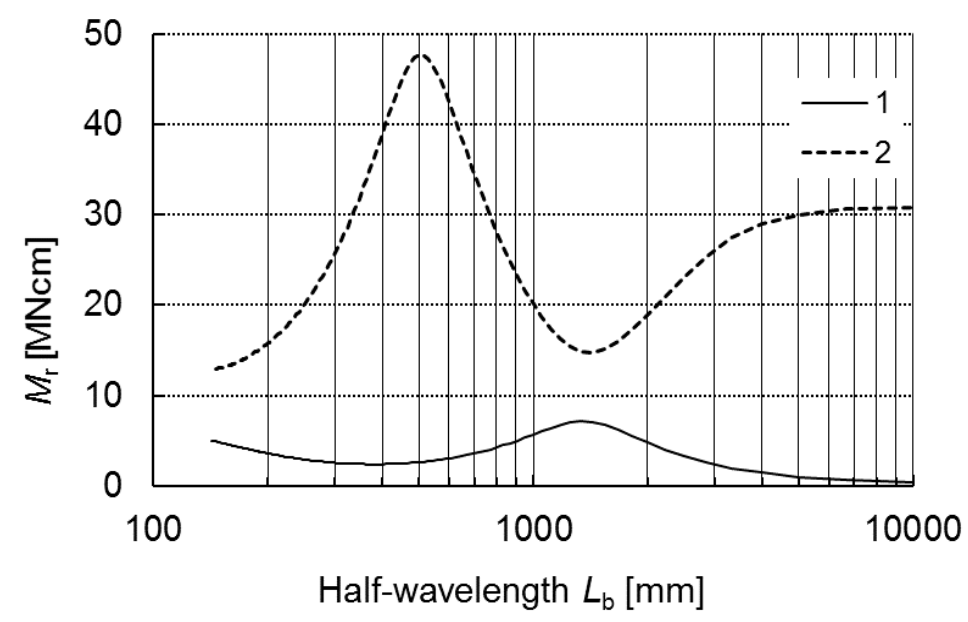

Figure 6. Buckling moments $M_{r}$ as a function of the buckling half-wavelength $L_{b}$ for LC-2.

Curve 1 corresponds to the lowest critical values of the bending moment, i.e., the primary buckling moments, whereas curve 2 corresponds to the secondary buckling moments. Curve 1 decreases in the range $100 \leq L_{b} \leq 400 \mathrm{~mm}$, and then it increases up to the maximal value at $L_{b}=1500 \mathrm{~mm}$. For higher lengths $L_{b}$, the critical moment decreases monotonously. On the other hand, curve 2 grows monotonously for $100 \leq L_{b} \leq 500 \mathrm{~mm}$ to attain its maximal value at $L_{b}=500 \mathrm{~mm}$. At $500 \leq L_{b} \leq$ $1500 \mathrm{~mm}$, it decreases sharply to grow next in the range $1500 \leq L_{b} \leq 4000 \mathrm{~mm}$, and then the critical values remain constant for $L_{b} \geq 4000 \mathrm{~mm}$ in point of fact.

To sum up, curve 1 attains its local minimum at $L_{b} \approx 400 \mathrm{~mm}$, curve 2 has its maximum at $L_{b} \approx$ $500 \mathrm{~mm}$, curve 1 attains its maximum and curve 2 its minimum at $L_{b} \approx 1500 \mathrm{~mm}$.

In Table 3 the results for the assumed four total lengths of LC- 2 beams are collected. The index notations were the same as in example 3.1 (LC-1). For the assumed lengths, values $M_{1}$ are lower and are actually the same except for the shortest beam of the length $L=250 \mathrm{~mm}$. At $L=250 \mathrm{~mm}$ and $L=400 \mathrm{~mm}$, the moment $M_{1}$ corresponds to the number of half-waves $m=1$. Thus, as for LC- 1 , the values of $M_{2}$ corresponding to the global mode are not given. On the other hand, the values of $M_{3}$ for the secondary mode, at which $m=1$, are given. For the assumed lengths $L$, we have $M_{3} / M_{1}>6$, and, for two longest beams, it is $M_{2} / M_{1} \geq 1.5$. Hence, for the lengths under consideration, sensitivity to imperfections decreases in comparison to example 3.1 (LC-1).

Table 3. The buckling moments $M_{r}$ with the corresponding number of half-waves $m$ of buckling along the longitudinal direction of the LC-2 and the dimensionless ratio of load carrying capacities $M_{s} / M_{\min }$ for different lengths $L$.

\begin{tabular}{cccccc}
\hline $\boldsymbol{L}$ & $\boldsymbol{M}_{\mathbf{1}}$ & $\boldsymbol{M}_{\mathbf{2}}$ & $\boldsymbol{M}_{\mathbf{3}}$ & $\boldsymbol{M}_{\boldsymbol{s} \mathbf{1}} / \boldsymbol{M}_{\min }$ & $\boldsymbol{M}_{\mathbf{s} 2} / \boldsymbol{M}_{\min }$ \\
\hline $\mathbf{M m}$ & $\mathbf{M N c m}$ & $\mathbf{M N c m}$ & $\mathbf{M N c m}$ & - & - \\
\hline 2000 & $2.413(5)$ & 4.807 & 18.94 & 0.803 & 0.816 \\
\hline 700 & $2.423(2)$ & 3.625 & 34.48 & 0.867 & 1.077 \\
\hline 400 & $2.413(1)$ & - & 38.91 & - & - \\
\hline 250 & $2.913(1)$ & - & 20.13 & - & - \\
\hline
\end{tabular}

In Figure 7a-d, buckling modes for LC-2 are shown. The local buckling mode (mode 1) for the four assumed lengths is the same. 


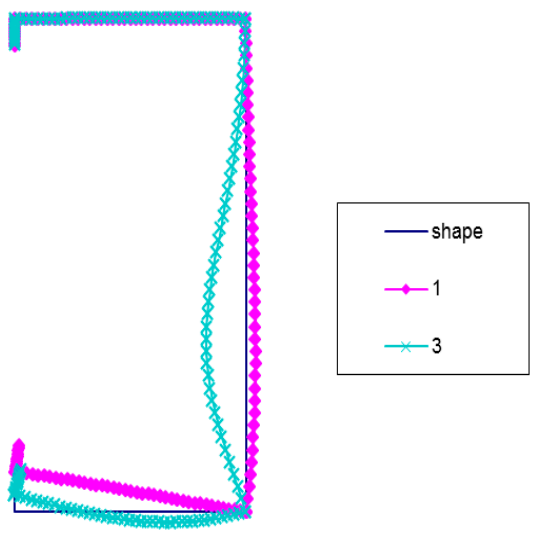

(a)

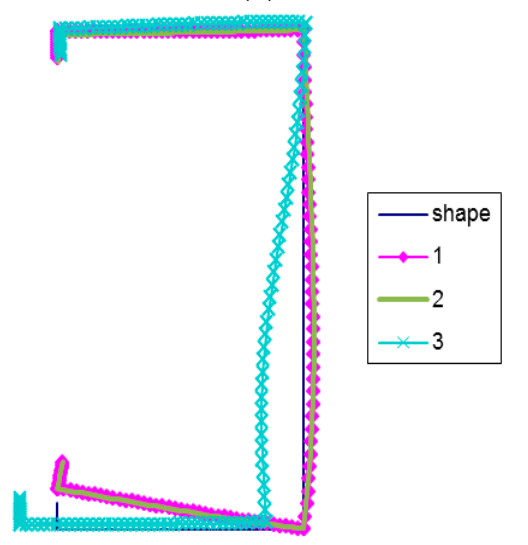

(c)

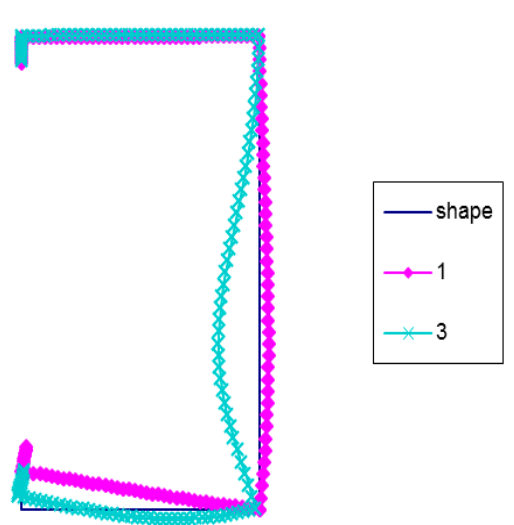

(b)

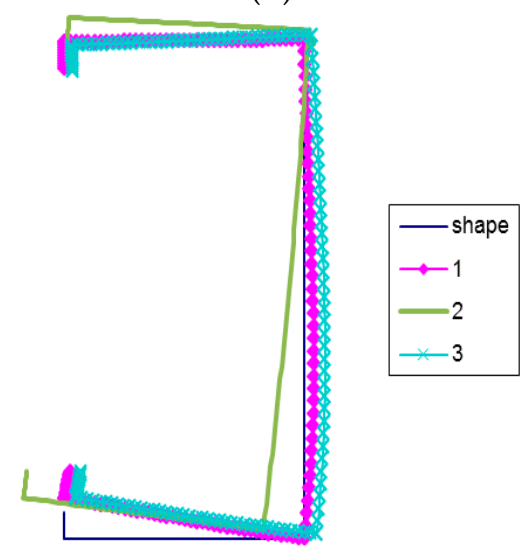

(d)

Figure 7. (a) Buckling modes for LC-2 beam lengths $L=250 \mathrm{~mm}$, (b) Buckling modes for LC-2 beam lengths $L=400 \mathrm{~mm}$, (c) Buckling modes for LC-2 beam lengths $L=700 \mathrm{~mm}$, (d) Buckling modes for LC-2 beam lengths $L=2000 \mathrm{~mm}$.

The deflection maximum lies in the corner of the compressed flange and reinforcement. The secondary global mode (mode 3 ) for $L=250 \mathrm{~mm}$ and $L=400 \mathrm{~mm}$ is the same. The maximal deflection takes place in the web. At $L=2000 \mathrm{~mm}$, mode 3 is similar to the local mode. Additionally, only displacements of the corner connecting the web with the flange under compression can be observed. At $L=700 \mathrm{~mm}$ and mode 3, the maximal deflection of the web is slightly higher than the displacement of lower corners. For this length, the global mode (curve 2) is identical to the local mode (curve 1), while for $L=2000 \mathrm{~mm}$, mode 2 corresponds to the distortional-lateral buckling mode, as there are no right angles in lower corners. Thus, all buckling modes (curves 1,2,3) are distortional modes.

In Table 3, the values of the ratio of the limit load carrying capacity to the minimal critical value for two- $(J=2)$ and three- $(J=3)$ mode approaches, $M_{s 2} / M_{\min }$ and $M_{\mathrm{s} 1} / M_{\min }$, respectively, are given. Like in example 3.1, the same values of imperfections were assumed.

For the lengths $L=250 \mathrm{~mm}$ and $L=400 \mathrm{~mm}$, limit values were not attained. For these lengths as for LC-1, it was assumed that $\zeta_{3}^{*}=|0.1|$, as $m=1$. For the remaining two lengths, the limit load carrying capacity is lower for the three-mode approach than for the two-mode approach, identically as for LC-1.

In the two next figures (Figures 8 and 9), a relationship of $M / M_{\min }$ versus the angle $\alpha / \alpha_{\min }$ is presented according to formula (A6) for the length $L=250 \mathrm{~mm}$ and $L=400 \mathrm{~mm}$. 


\section{LC-2 L=250mm}

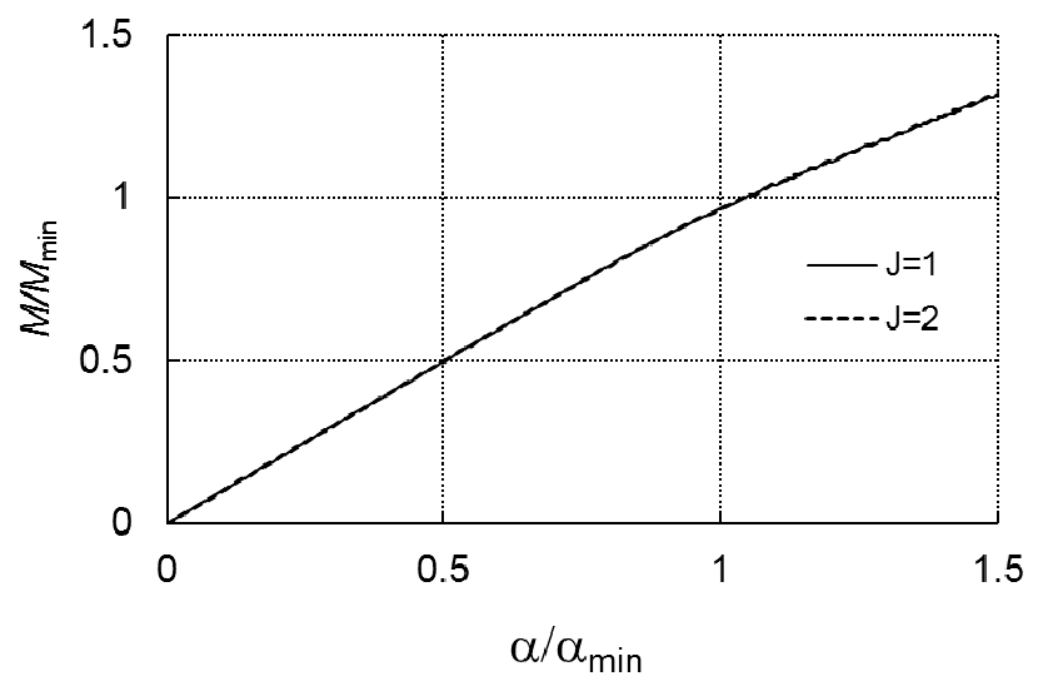

Figure 8. $M / M_{\min }$ versus $\alpha / \alpha_{\min }$ for LC-2 and $L=250 \mathrm{~mm}$.

LC-2 L $=400 \mathrm{~mm}$

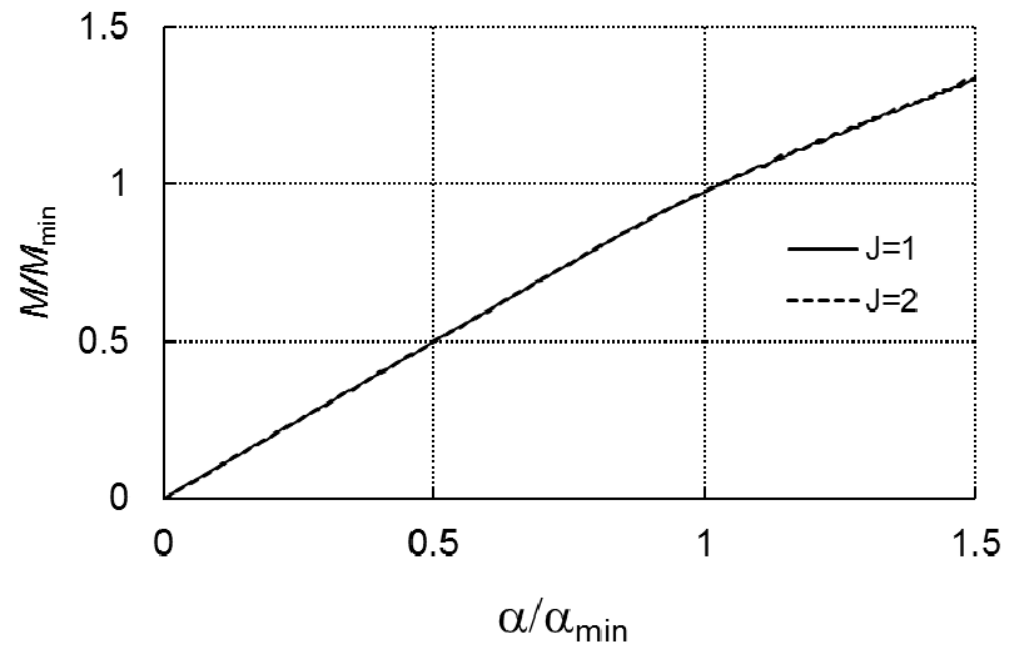

Figure 9. $M / M_{\min }$ versus $\alpha / \alpha_{\min }$ for LC-2 and $L=400 \mathrm{~mm}$.

Curve 1 corresponds to the case of one-mode buckling $(r=J=1)$, while curve 2 corresponds to two-mode buckling $(J=2, r=1, r=3)$. Both the curves overlap, which proves a lack of an interaction between the modes in the range of loading under consideration. The dependence of $\alpha / \alpha_{\min }$ on $M / M_{\min }$ at $L=700 \mathrm{~mm}$, for the two- $(J=2)$ and three-mode $(J=3)$ approach, correspondingly, is presented in Figure 10. In the case of $J=3$, the limit load carrying capacity is $M_{s 1} / M_{\min }=0.867$, whereas, for $J=2$, $M_{\mathrm{s} 2} / M_{\min }$ cannot be determined.

In this case, a significant effect of the secondary global mode $(r=3)$ on the load carrying capacity can be seen. At $L=2000 \mathrm{~mm}$, the quantities $M_{s 1} / M_{\min }$ and $M_{s 2} / M_{\min }$ differ slightly, i.e., by less than $2 \%$.

For $L=250 \mathrm{~mm}$ and $L=400 \mathrm{~mm}$, the nonlinear coefficients (A3) $\zeta_{1}^{2} \zeta_{3}$, responsible for the interaction of modes, are very low and, moreover, $M_{3} / M_{1}>6$; thus, we encounter one-mode buckling for the loads $M / M_{\min }$ under analysis. At $L=700 \mathrm{~mm}$, the terms including the coefficients $\zeta_{1}^{2} \zeta_{3}, \zeta_{2}^{2} \zeta_{3}, \zeta_{3}^{2} \zeta_{2}$ play an important role, whereas, at $L=2000 \mathrm{~mm}$, the terms are $\zeta_{1}^{2} \zeta_{2}, \zeta_{1}^{2} \zeta_{3}$.

In Reference [19], for the length $L=2000 \mathrm{~mm}$, the dimensional load carrying capacity is equal to $M_{s} / M_{\min }=0.919$, and, in this work, it is $M_{s 1} / M_{\min }=0.803$. One should remember that the values of global imperfections were assumed differently. At $L=400 \mathrm{~mm}$ in Reference [19], the load carrying capacity was not determined either. 
LC-2 L=700mm

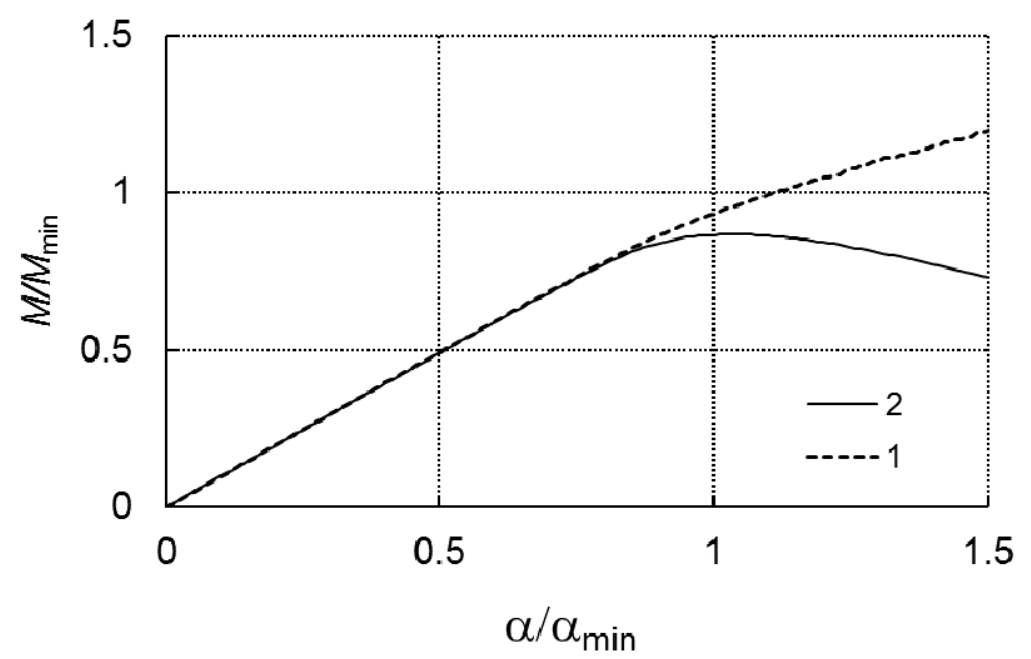

Figure 10. $M / M_{\min }$ versus $\alpha / \alpha_{\min }$ for LC-2 and $L=700 \mathrm{~mm}$.

\subsection{Example of LC-3 Beams}

Like in earlier examples, detailed geometrical dimensions are listed in Table 2. In Figure 11, alternations in critical bending moments $M_{r}$ as a function of the buckling half-wavelength $L_{b}$ are presented.

LC-3

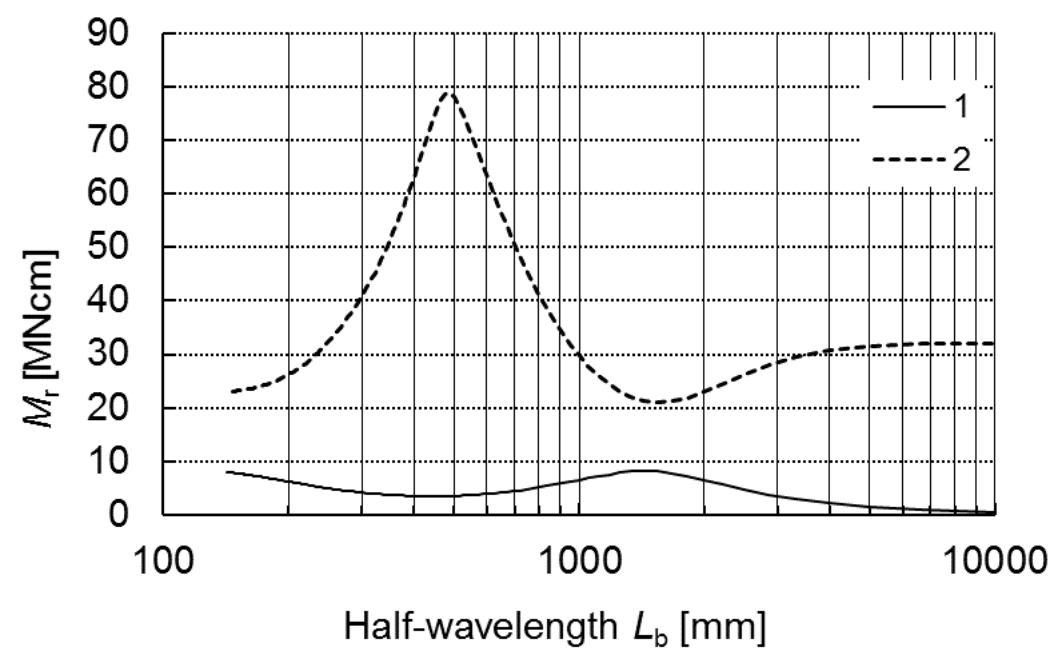

Figure 11. Buckling moments $M_{r}$ as a function of the buckling half-wavelength $L_{b}$ for LC-3.

Curve 1 corresponds to the lowest values of the critical moment, while curve 2 corresponds to higher values for $100 \leq L_{b} \leq 10,000 \mathrm{~mm}$. The plots of both curves are similar to the plots in Figure 2 (LC-1) and Figure 6 (LC-2). The minimal local value of the moment for curve 1 was attained at $L_{b} \approx$ $450 \mathrm{~mm}$, the local maximum was attained at $L_{b} \approx 1500 \mathrm{~mm}$, whereas curve 2 attains the maximal value of the moment for $L_{b} \approx 480 \mathrm{~mm}$ and the minimal value for $L_{b} \approx 1500 \mathrm{~mm}$, respectively.

As in former examples, Table 4 lists values of critical loads for 4 selected lengths of beams $L$. At $L=300 \mathrm{~mm}$, the lowest local mode $M_{1}$ occurs for $m=1$. Thus, mode 2 was not considered. The value $M_{3}$ (for $m=1$ ) is almost 10-times higher than $M_{1}$. At $L=800 \mathrm{~mm}$, we have $M_{2} / M_{1}=1.4$, and for $L=2500 \mathrm{~mm}$ it is $M_{2} / M_{1}=1.3$, whereas, at $L=4500 \mathrm{~mm}$, the global value $M_{2}$ is lower than $M_{1}$, as $M_{2} / M_{1}=0.5$. The secondary value of $M_{3}$ for the values of $L$ under analysis is at least 7-times higher than $M_{1}$. 
Table 4. The buckling moments $M_{r}$ with the corresponding number of half-waves $m$ of buckling along the longitudinal direction of the LC-3 and the dimensionless ratio of load carrying capacities $M_{s} / M_{\text {min }}$ for different lengths $L$.

\begin{tabular}{cccccc}
\hline $\boldsymbol{L}$ & $\boldsymbol{M}_{\mathbf{1}}$ & $\boldsymbol{M}_{\mathbf{2}}$ & $\boldsymbol{M}_{\mathbf{3}}$ & $\boldsymbol{M}_{\boldsymbol{s} \mathbf{1}} / \boldsymbol{M}_{\min }$ & $\boldsymbol{M}_{\boldsymbol{s} \mathbf{2}} / \boldsymbol{M}_{\min }$ \\
\hline $\mathbf{m m}$ & $\mathbf{M N c m}$ & $\mathbf{M N c m}$ & $\mathbf{M N c m}$ & - & - \\
\hline 4500 & $3.499(10)$ & 1.765 & 31.23 & 0.770 & 0.774 \\
\hline 2500 & $3.515(6)$ & 4.686 & 26.27 & 0.753 & 0.763 \\
\hline 800 & $3.544(2)$ & 5.105 & 41.15 & 0.833 & - \\
\hline 300 & $4.174(1)$ & - & 40.87 & - & - \\
\hline
\end{tabular}

In Figure 12a-d, buckling modes for selected lengths $L$ are presented. Local buckling modes (mode 1) are practically the same for all lengths.

At $L=300 \mathrm{~mm}$ and mode $3(m=1)$, maximal deflections occur in the web. At $L=800 \mathrm{~mm}$, also the global mode (mode 2 ) is identical to mode 1 (Figure 12b). The secondary global mode (mode 3 ) has the maximal deflection for lower compressed corners of LC-3. Mode 2 (curve 2) for the length $L=2500 \mathrm{~mm}$ and $L=4500 \mathrm{~mm}$ is a "pure" lateral buckling mode in principle. At $L=2500 \mathrm{~mm}$ and mode 3, a slight displacement of the corner connecting the web with the compressed lower flange takes place, whereas, for $L=4500 \mathrm{~mm}$, displacements of both web corners occur.

Moreover, Table 4 also shows the dimensionless limit load carrying capacity for two- and three-mode approaches, $M_{s 2} / M_{\min }$ and $M_{s 1} / M_{\min }$, respectively. At $L=2500 \mathrm{~mm}$ and $L=4500 \mathrm{~mm}$, differences between both the approaches are inconsiderable.

In Figure 13, at the length $L=300 \mathrm{~mm}$, curve 1 for the one-mode approach $(J=1)$ overlaps curve 2 for the two-mode approach $(J=2, r=1, r=3)$.

For the range of loadings under consideration, there is no interaction between the modes. For $L=800 \mathrm{~mm}$, in the case of the three-mode, we have $M_{s 1} / M_{\min }=0.833$, whereas, for the two-mode approach, there is no limit load carrying capacity (Figure 14).

For $L=300 \mathrm{~mm}$, the value of the coefficient at the term $\zeta_{1}^{2} \zeta_{3}$ is inconsiderable, but at $L=800 \mathrm{~mm}$, the terms $\zeta_{1}^{2} \zeta_{3}, \zeta_{2}^{2} \zeta_{3}$ play a significant role. At $L=2500 \mathrm{~mm}$ and at $L=4500 \mathrm{~mm}$, the coefficients at the terms $\zeta_{1}^{2} \zeta_{2}, \zeta_{1}^{2} \zeta_{3}$ are important.

For the length $L=4500 \mathrm{~mm}$ in Reference [19], the value of the load carrying capacity was $M_{s} / M_{\min }=0.806$, whereas, in the present analysis, it was $M_{s 1} / M_{\min }=0.77$.

For all the examples under analysis in Reference [19], higher values were attained than in the present study. One should note once more that the values of local imperfections in Reference [19] were assumed in a different way than here.

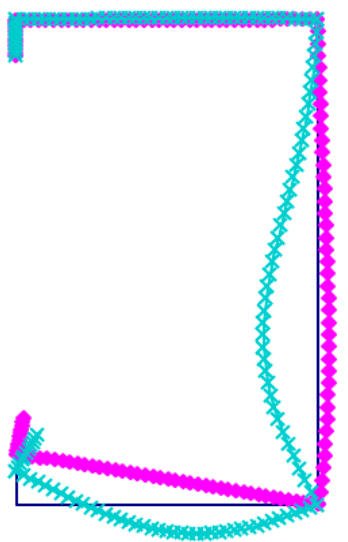

(a)

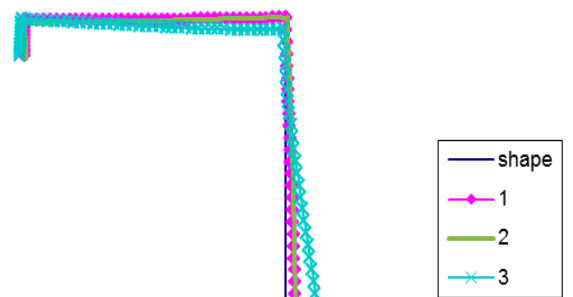

(b)

Figure 12. Cont. 


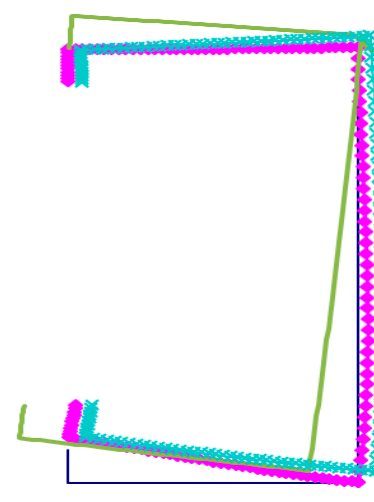

(c)

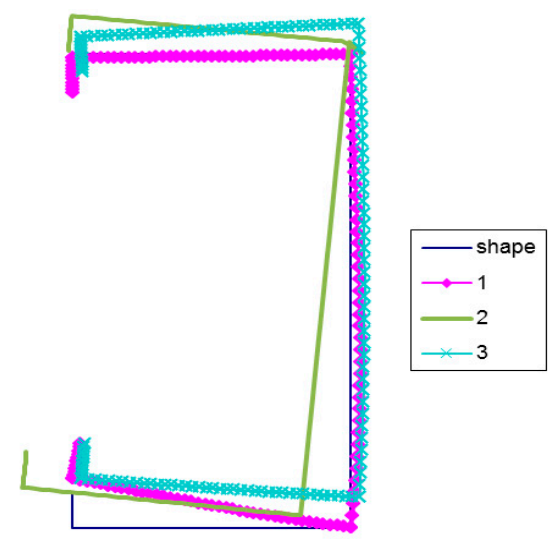

(d)

Figure 12. (a) Buckling modes for LC-3 beam lengths $L=300 \mathrm{~mm}$, (b). Buckling modes for LC-3 beam lengths $L=800 \mathrm{~mm}$, (c) Buckling modes for LC-3 beam lengths $L=2500 \mathrm{~mm}$, (d) Buckling modes for LC-3 beam lengths $L=4500 \mathrm{~mm}$.

\section{LC-3 $L=300 \mathrm{~mm}$}

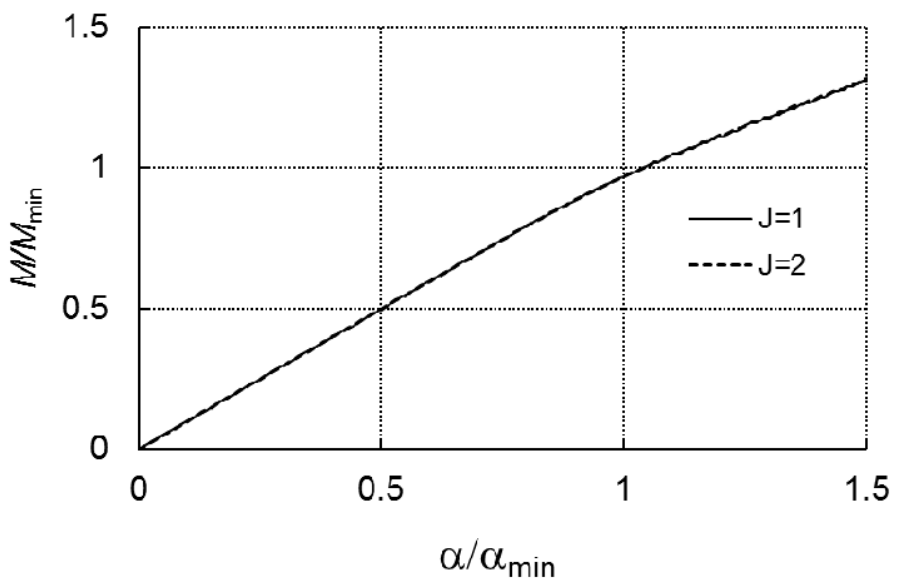

Figure 13. $M / M_{\min }$ versus $\alpha / \alpha_{\min }$ for LC-3 and $L=300 \mathrm{~mm}$.

CC-3 $L=800 \mathrm{~mm}$

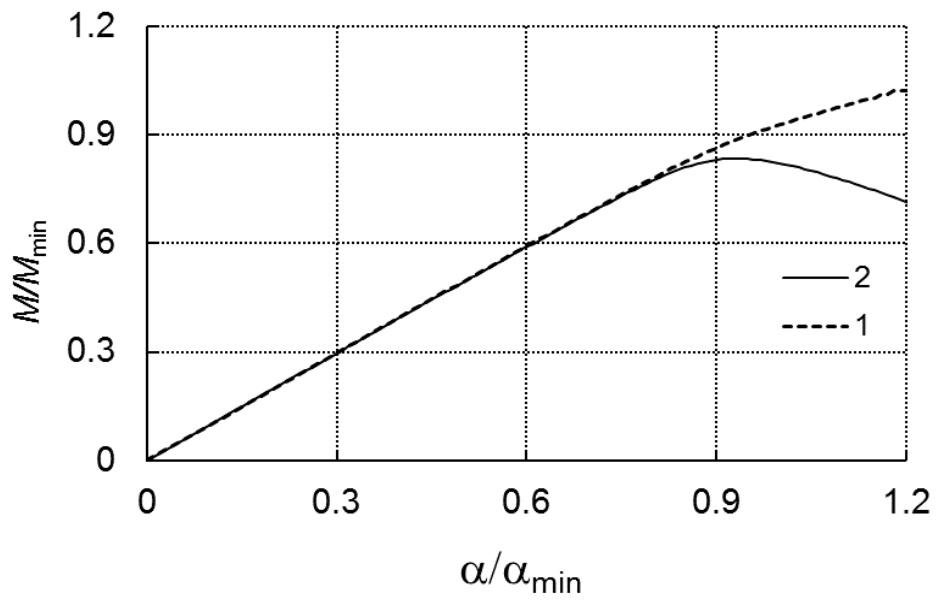

Figure 14. $M / M_{\min }$ versus $\alpha / \alpha_{\min }$ for LC-3 and $L=800 \mathrm{~mm}$. 
The plots of variability in critical moments (curves 1 and 2) as a function of the half-wavelength $L_{b}$ shown in Figures 2, 6 and 11 as well as an analysis of buckling modes, the load carrying capacity and the effect of nonlinear coefficients at the first-order approximation terms allow one to classify, according to the conclusions expressed in Reference [13], the following lengths of LC-beams, namely:

(1) short beams (LC- $1-100 \leq L_{b} \leq 350 \mathrm{~mm}$; LC-2 - $100 \leq L_{b} \leq 500 \mathrm{~mm}$; LC-3 $-100 \leq L_{b} \leq 450 \mathrm{~mm}$ );

(2) medium-long beams (LC- $1-350 \leq L_{b} \leq 1050 \mathrm{~mm}$; LC-2 $-500 \leq L_{b} \leq 1500 \mathrm{~mm}$; LC-3 - $450 \leq L_{b} \leq$ $1050 \mathrm{~mm}$ );

(3) long beams (LC-1 - $1050 \leq L_{b} \leq 3500 \mathrm{~mm}$; LC-2 - $1500 \leq L_{b} \leq 4000 \mathrm{~mm}$; LC-3 - $1050 \leq L_{b} \leq$ $4000 \mathrm{~mm})$;

(4) very long beams (LC-1 - $3500 \mathrm{~mm} \leq L_{b}$; LC-2 - $4000 \mathrm{~mm} \leq L_{b}$; LC-3 $-4000 \mathrm{~mm} \leq L_{b}$ ).

Compared to Reference [13], the term of very long beams, for which the secondary global mode $M_{3}$ is actually constant and the primary global mode $M_{2}$ has a low gradient of the value drop in comparison to long beams, is introduced additionally in the above-mentioned classification.

Particular attention was paid to the influence of secondary global distortional-lateral buckling mode on the load carrying capacity for the LC-beams under bending. As demonstrated in the paper, the most significant influence is for medium-long beams. In this case, disregarding the interaction of three modes, including two global (i.e., primary and secondary) and local ones, may lead to an incorrect assessment of the load carrying capacity of the two-mode approach for medium-long beams.

\section{Conclusions}

The stability and interactive buckling of steel LC-beams under bending, for three different cross-sections in a wide range of beam length variability, were investigated. Attention was paid in particular to an effect of the secondary global buckling mode on an interaction between modes, including distortional buckling modes. A classification of lengths of beams subjected to bending in the web plane, starting from short to medium-long up to long, or even very long ones, is proposed. In the cases under consideration, the influence of the secondary global mode on the load carrying capacity is most evident for medium-long beams. It is advisable to extend this analysis onto an effect of the length of edge reinforcements and the wall thickness of LC-beams on the interactive buckling and load carrying capacity. It is important to conduct validation of the described and analyzed phenomenon, using the semi-analytical method SAM through the use of more comprehensive modeling using FEM.

Author Contributions: Conceptualization, Z.K.; Methodology, Z.K.; Software, Z.K.; Validation, J.J.; Formal Analysis, Z.K. and J.J.; Investigation, Z.K.; Resources, J.J.; Data Curation, Z.K.; Writing-Original Draft Preparation, Z.K.; Writing-Review \& Editing, J.J.; Visualization, J.J.; Supervision, Z.K.; Project Administration, Z.K.

Funding: This study was financially supported with the Research Project No. UMO-2017/25/B/ST8/00007 granted by the National Science Centre, Poland.

Conflicts of Interest: The authors declare no conflict of interest.

\section{Appendix A}

For each plate component, precise geometrical relationships (i.e., full Green's strain tensor) were assumed in order to consider both out-of-plane and in-plane bending of the $i$-th plate [11-14]:

$$
\begin{aligned}
& \varepsilon_{x i}=u_{i, x}+\frac{1}{2}\left(w_{i, x}^{2}+v_{i, x}^{2}+u_{i, x}^{2}\right) \\
& \varepsilon_{y i}=v_{i, y}+\frac{1}{2}\left(w_{i, y}^{2}+u_{i, y}^{2}+v_{i, y}^{2}\right) \\
& 2 \varepsilon_{x y i}=\gamma_{x y i}=u_{i, y}+v_{i, x}+w_{i, x} w_{i, y}+u_{i, x} u_{i, y}+v_{i, x} v_{i, y}
\end{aligned}
$$

and

$$
\kappa_{x i}=-w_{i, x x} \kappa_{y i}=-w_{i, y y} \kappa_{x y i}=-2 w_{i, x y},
$$

where: $u_{i}, v_{i}, w_{i}$-components of the displacement vector of the $i$-th plate along the $x_{i}, y_{\mathrm{i}}, z_{i}$ axis direction, respectively, and the plane $x_{i}-y_{\mathrm{i}}$ overlaps the central plane before its buckling. 
The nonlinear problem of stability was solved with Koiter's theory [11-14]. The displacement fields $U$ and the sectional force fields $N$ were expanded into power series with respect to the dimensionless amplitude of the $r$-th mode deflection $\zeta_{r}$.

For thin-walled structures with the geometric imperfections $\bar{U}$ (only the linear initial imperfections determined by the shape of the $r$-th buckling modes, i.e., $\left.\bar{U}=\zeta_{r}^{*} U_{r}\right)$, the total potential energy has the form:

$$
\Pi=-\frac{1}{2} M^{2} \bar{a}_{0}+\frac{1}{2} \sum_{r=1}^{J} \bar{a}_{r} \zeta_{r}^{2}\left(1-\frac{M}{M_{r}}\right)+\frac{1}{3} \sum_{p}^{J} \sum_{q}^{J} \sum_{r}^{J} \bar{a}_{p q r} \zeta_{p} \zeta_{q} \zeta_{r}+\frac{1}{4} \sum_{r}^{J} \bar{b}_{r r r r} \zeta_{r}^{3}-\sum_{r}^{J} \frac{M}{M_{r}} \bar{a}_{r} \zeta_{r}^{*} \zeta_{r},
$$

then, the equilibrium equations corresponding to (A3) are as follows:

$$
\left(1-\frac{M}{M_{r}}\right) \xi_{r}+a_{p q r} \xi_{p} \xi_{q}+b_{r r r r} \xi_{r}^{3}=\frac{M}{M_{r}} \xi_{r}^{*}, r=1, \ldots, J
$$

where, $M$ is a magnitude of the applied bending moment, $M_{r}, \zeta_{r}, \zeta_{r}^{*}$ is the buckling moment of the $r$-th buckling mode, the dimensionless amplitude of the $r$-th buckling mode and the dimensionless amplitude of the initial deflection corresponding to the $r$-th buckling mode, respectively. The buckling modes $U_{i}$ are mutually orthogonal in the following sense $\sigma_{0} \cdot l_{11}\left(U_{I}, U_{K}\right)=0,(I, K)=[1, J], I \neq K$ where $J$ is all the relevant buckling modes that are believed to be important in the structural response. Bifurcation mode imperfections can have positive and negative quantities. The signs of imperfections were chosen for the most unfavorable fashion case (i.e., load carrying capacity would assume its minimum value). The coefficients $\bar{a}_{0}, \bar{a}_{r}, \bar{a}_{p q r}$ and $\bar{b}_{r r r r}$ can be determined with the equations described in the literature $[11,12,14]$.

The following notations are introduced in (4):

$$
a_{p q r}=\bar{a}_{p q r} / \bar{a}_{r} b_{r r r r}=\bar{b}_{r r r r} / \bar{a}_{r}
$$

In the semi-analytical method (SAM), one postulates to determine approximated values of the $b_{r r r r}$ coefficients (A4) on the basis of the linear buckling problem. This approach allows the values of the $a_{p q r}$ coefficients (A4) to be precisely determined, according to the applied nonlinear Byskov and Hutchinson theory [11,12].

A relative angle of rotation of the girder in bending on the support as a function of the $M / M_{\text {min }}$ load was determined through differentiation of the expression for potential energy (A3) with respect to $M / M_{\min }[11,12,14]$ :

$$
\frac{\alpha}{\alpha_{\min }}=\frac{M}{M_{\min }}\left[1+\frac{M_{\min }}{M \bar{a}_{0}} \sum_{r=1}^{J} \frac{M_{\min }}{M_{r}} \bar{a}_{r} \zeta_{r}\left(0.5 \zeta_{r}+\zeta_{r}^{*}\right)\right],
$$

where $\alpha_{\min }$ is the minimal critical angle of rotation of the beam under pure bending, corresponding to the minimal value of the critical moment $M_{\min }$.

At the point where the load parameter $M$ reaches its maximum value $M_{S}$ (the so-called theoretical load carrying capacity) for the initial geometrical imperfect structure with the amplitude $\zeta_{r}^{*}$, the Jacobian of the nonlinear system of Equation (A4) is equal to zero.

\section{References}

1. Abambres, M.; Camotim, D.; Silvestre, N. Modal decomposition of thin-walled member collapse mechanisms. Thin-Walled Struct. 2014, 74, 269-291. [CrossRef]

2. Adany, S. Constrained shell Finite Element Method for thin-walled members, Part 1: constrains for a single band of finite elements. Thin-Walled Struct. 2018, 128, 43-55. [CrossRef]

3. Adany, S.; Visy, D.; Nagy, R. Constrained shell Finite Element Method, Part 2: application to linear buckling of thin-walled members. Thin-Walled Struct. 2018, 128, 56-70. [CrossRef] 
4. Adany, S. Modal identification of thin-walled members by using the constrained finite element method. Thin-Walled Struct. 2019, 140, 31-42. [CrossRef]

5. Bebiano, R.; Camotim, D.; Gonçalves, R. GBTUL 2.0-A second-generation code for the GBT-based buckling and vibration analysis of thin-walled members. Thin-Walled Struct. 2018, 124, 235-257. [CrossRef]

6. Camotim, D.; Dinis, P.B.; Martins, A.D.; Young, B. Review: Interactive behaviour, failure and DSM design of cold-formed steel members prone to distortional buckling. Thin-Walled Struct. 2018, 128, 12-42. [CrossRef]

7. Garcea, G.; Leonetti, L.; Magisano, D.; Goncalves, R.; Camotim, D. Deformation modes for the post-critical analysis of thin-walled compressed members by a Koiter semi-analytic approach. Int. J. Solids Struct. 2017, 110-111, 367-384. [CrossRef]

8. Gliszczyński, A.; Kubiak, T. Load-carrying capacity of thin-walled composite beams subjected to pure bending. Thin-Walled Struct. 2017, 115, 76-85. [CrossRef]

9. Hancock, G.J. Coupled Instabilities in Metal Structures (CIMS) —What have we learned and are we going? Thin-Walled Struct. 2018, 128, 2-11. [CrossRef]

10. Jakubczak, P.; Gliszczynski, A.; Bienias, J.; Majerski, K.; Kubiak, T. Collapse of channel section composite profile subjected to bending, Part II: Failure analysis. Compos. Struct. 2017, 179, 1-20. [CrossRef]

11. Kolakowski, Z.; Krolak, M. Modal coupled instabilities of thin-walled composite plate and shell structures. Compos. Struct. 2006, 76, 303-313. [CrossRef]

12. Kolakowski, Z.; Mania, J.R. Semi-analytical method versus the FEM for analysis of the local post-buckling. Compos. Struct. 2013, 97, 99-106. [CrossRef]

13. Kolakowski, Z.; Jankowski, J. Interactive buckling of steel C-beams with different lengths-From short to long beams. Thin-Walled Struct. 2018, 125, 203-210. [CrossRef]

14. Kołakowski, Z.; Urbaniak, M. Influence of the distortional-lateral buckling mode on the interactive buckling of short channels. Thin-Walled Struct. 2016, 109, 296-303. [CrossRef]

15. Kubiak, T.; Kolakowski, Z.; Swiniarski, J.; Urbaniak, M. Local buckling and post-buckling of composite channel-section beams-Numerical and experimental investigations. Compos. Struct. Part B 2016, 91, 176-188. [CrossRef]

16. Martins, A.D.; Landesmann, A.; Camotim, D.; Dinis, P.B. Distortional failure of cold-formed steel beams under uniform bending: Behaviour, strength and DSM design. Thin-Walled Struct. 2017, 118, 196-213. [CrossRef]

17. Martins, A.D.; Camotim, D.; Dinis, P.B. Local-distortional interaction in cold-formed steel beams: Behaviour, strength and DSM design. Thin-Walled Struct. 2017, 119, 879-901. [CrossRef]

18. Martins, A.D.; Camotim, D.; Dinis, P.B. On the distortional-global interaction in cold-formed steel columns: Relevance, post-buckling behaviour, strength and DSM design. J. Constr. Steel Res. 2018, 145, 449-470. [CrossRef]

19. Martins, A.D.; Camotim, D.; Goncalves, R.; Dinis, P.B. GBT-based assessment of the mechanics of distortional-global interaction in thin-walled lipped channel beams. Thin-Walled Struct. 2018, 124, $32-47$. [CrossRef]

20. Martins, A.D.; Camotim, D.; Gonçalves, R.; Dinis, P.B. On the mechanics of local-distortional interaction in thin-walled lipped channel beams. Thin-Walled Struct. 2018, 128, 108-125. [CrossRef]

21. Martins, A.D.; Camotim, D.; Dinis, P.B. Distortional-global interaction in lipped channel and zed-section beams: Strength, relevance and DSM design. Thin-Walled Struct. 2018, 129, 289-308. [CrossRef]

22. Niu, S.; Rasmussen, K.J.R.; Fan, F. Distortional-global interaction buckling of stainless steel C-beams: Part I-Experimental investigation. J. Constr. Steel Res. 2014, 96, 127-139. [CrossRef]

23. Niu, S.; Rasmussen, K.J.R.; Fan, F. Distortional-global interaction buckling of stainless steel C-beams: Part II-Numerical study and design. J. Constr. Steel Res. 2014, 96, 40-53. [CrossRef]

24. Szymczak, C.; Kujawa, M. Buckling and initial post-local buckling behaviour of cold-formed channel member flange. Thin-Walled Struct. 2019, 137, 177-184. [CrossRef]

25. Yadav, K.K.; Gerasimidis, S. Instability of thin steel cylindrical shells under bending. Thin-Walled Struct. 2019, 137, 151-166. [CrossRef] 
26. Yang, K.; Yu, Z.-H. Experimental research on the creep buckling of fire-resistant steel columns at elevated temperature. Steel Compos. Struct. 2013, 15, 163-173. [CrossRef]

27. Zhang, X.; Peng, L.; Ni, Z.-P.; Ni, T.-X.; Huang, Y.-L.; Zhou, Y. Experimental study on the fire performance of tubular steel columns with membrane protections for prefabricated and modular steel construction. Materials 2018, 11, 437. [CrossRef] [PubMed] 